\title{
Nuevos registros del dinoflagelado Craspedotella Kofoid, 1905 (Dinophyceae: Noctilucales) en el Océano Pacífico
}

\author{
New records of the dinoflagellate Craspedotella Kofoid, 1905 \\ (Dinophyceae: Noctilucales) in the Pacific Ocean \\ Fernando Gómez ${ }^{1}$ \\ ${ }^{1}$ Station Marine de Wimereux, Université des Sciences et Technologies de Lille-Lille1, \\ FRE 2816 ELICO CNRS, 28 avenue Foch, BP 80, F-62930 Wimereux, Francia \\ fernando.gomez@fitoplancton.com
}

\begin{abstract}
Specimens assigned to the dinoflagellate genus Craspedotella Kofoid 1905 are reported for the first time after the initial description in the Eastern Pacific. Craspedotella sp. is composed of a dome with radial fibrils in the lower margin and a broad band at the base with unidentified structures, tentatively pores. Another specimen, identified as Craspedotella cf. pileolus, was smaller than the type species. Other
\end{abstract}

specimens with a conical shape and a pointed apex have been assigned to the genus. The morphological discrepancies in the Kofoid's description and later by Cachon \& Cachon make difficult the identification of Craspedotella pileolus.

Key words: Craspedotella pileolus, Leptodiscus, Leptodiscaceae, Dinophyta, Pacific Ocean
Los dinoflagelados del orden Noctilucales Haeckel divergen de la morfología más común de estos organismos. El orden esta constituido por las familias Noctilucaceae Saville-Kent, Kofoidiniaceae F.J.R. Taylor y Leptodiscaceae Kofoid, siendo esta última familia la menos conocida. Las leptodiscáceas se caracterizan por un cuerpo celular cubierto de fibrillas, muy aplanado con simetría bilateral o radial, carecen de cíngulo y los flagelos pueden estar ausentes. Tras la descripción de la especie tipo Leptodiscus medusoides Hertwig en el Mediterráneo, Kofoid (1905) describió Craspedotella pileolus Kofoid en el Pacífico oriental y estableció la familia Leptodiscaceae. Posteriormente se describieron los otros géneros monotípicos Scaphodinium Margalef (=Leptospathium J. Cachon \& CachonEnjumet), Abedinium Loeblich \& A.R. Loeblich (=Leptophyllus J. Cachon \& Cachon-Enjumet), Petalodinium J. Cachon \& M. Cachon y Cachonodinium A.R. Loeblich (=Leptodinium J. Cachon \& M. Cachon). Los registros de leptodiscáceas han sido muy escasos, probablemente debido a que estos organismos son delicados y se deterioran con la fijación y manipulación de las muestras. Las descripciones de leptodiscáceas no aparecen en las guías de identificación y la información está casi restringida al estudio realizado por Cachon \& Cachon (1969). Estos factores, junto con una morfología que difiere de los típicos dinoflagelados, hacen que las leptodiscáceas pasen inadvertidas para los observadores.

Todos los géneros de leptodiscáceas se describieron en el Mediterráneo occidental, con la excepción de Craspedotella en el Pacífico sureste. Kofoid (1905) describió $C$. pileolus a partir de un espécimen recogido por la Expedición Albatross 1904-5 en las proximidades de las Islas Galápagos (157'S, $117^{\circ} 1^{\prime} \mathrm{W}$ ) y otro espécimen en la costa de San Diego, California. El autor no proporciona ninguna ilustración, ni en la descripción original, ni en una monografía posterior sobre dinoflagelados atecados (Kofoid \& Swezy 1921). Kofoid describió Craspedotella como una célula acampanada de sección circular con un diámetro de 150-180 $\mu \mathrm{m}$. El nombre del género viene dado por su semejanza a la forma de la medusa craspedota Halistaura (Laodicea) cellularia A. Agassiz. La morfología se asemeja a Leptodiscus medusoides, aunque Craspedotella presenta un velo horizontal perforado en su base. Kofoid describe las vainas de dos flagelos en la parte superior del domo y un citostoma. En las costas mediterráneas francesas, Cachon \& Cachon (1969) adscribieron algunos especímenes a Craspedotella pileolus, aunque con algunas diferencias que según esos autores no justificaban la creación de una nueva especie. Entre otras diferencias, los especímenes mediterráneos eran 
de un diámetro menor $(<120 \mu \mathrm{m})$, y ninguno tenía flagelos. Hasta mi conocimiento no existe ningún otro antecedente del género Craspedotella.

Las primeras citas de leptodiscáceas en el Océano Pacífico con los géneros Scaphodinium, Petalodinium y Leptodiscus han sido recientes (Gómez \& Furuya 2004, 2005). Durante esos estudios en el Pacífico noroeste no apareció ningún espécimen que pudiese adscribirse a Craspedotella. Posteriormente en una campaña oceanográfica en el Pacífico sureste se observaron especímenes que se asemejan a la descripción de Kofoid (1905), aunque la falta de ilustración en ésta dificulta la identificación. Los muestras se tomaron durante la campaña francesa BIOSOPE a bordo del B/O L'Atalante entre las Islas Marquesas y la costa chilena a la altura de Concepción. Las muestras fijadas con solución ácida de Lugol se analizaron con un microscopio invertido Olympus y los especímenes se fotografiaron a 400 aumentos. También se incluye un espécimen procedente del Pacífico noroeste que se analizó siguiendo la metodología descrita en Gómez \& Furuya $(2004,2005)$.

El espécimen que se aproxima más a Craspedotella por su mayor tamaño fue recogido a 60 metros de profundidad en las proximidades del Archipiélago de Juan Fernández (Tabla 1). La célula con una sección circular de $132 \mu \mathrm{m}$ de diámetro estaba constituida por un domo sobre una banda con extremos que se inclinan hacia una base más reducida. El núcleo estaba localizado en una depresión en la parte superior del domo, en una posición ligeramente excéntrica (Fig. 1).
No se ha podido observar ningún flagelo, aunque no debe descartarse su presencia ya que el flagelo es una estructura muy delicada que suele perderse con el tratamiento de la muestra. En la parte inferior del domo aparecen unas fibrillas radiales que no llegan al límite con la banda inferior. Las fibrillas, separadas unas de otras unos $15 \mu \mathrm{m}$, se ramifican en su base (Fig. 2). En la banda inferior, entre cada dos fibrillas, aparece una estructura alargada y oblicua a la base, tentativamente poros, aunque no pudo ser observada con mayor resolución debido a las limitaciones ópticas (Fig. 3).

Otras células cercanas en forma a Craspedotella son de menor tamaño, entre 40 y $76 \mu \mathrm{m}$ en diámetro (Tabla 1). Un espécimen recogido en las proximidades de las Islas Marquesas presentaba una morfología similar a Craspedotella, pero con un diámetro sólo $52 \mu \mathrm{m}$ (Fig. 4). El núcleo tenía una forma elipsoidal de $17 \mu \mathrm{m}$ de longitud (Figs. 5, 6). En la región de la banda inferior aparecen fibrillas o aparentemente vellosidades (Fig. 4). Otro espécimen de menor tamaño presentaba una espina apical corta y un núcleo de contorno circular (Fig. 7). Tres especímenes se caracterizaban por una forma cónica con su ápice ligeramente excéntrico culminado por una espina más o menos desarrollada (Figs. 8-13). En su base presentaban un velo con un orificio de contorno circular (Fig. 13). En un espécimen recogido en el norte del Mar de las Filipinas y analizado con mejores recursos ópticos se podían observar numerosas vacuolas y alrededor de la banda inferior unas vellosidades que podrían corresponder a cilios (Fig. 10).

Tabla 1

Coordenadas geográficas, profundidad (m) y diámetro celular $(\mu \mathrm{m})$ de los registros de Craspedotella

Geographic coordinates, depth (m) and cell diameter $(\mu \mathrm{m})$ of the records of Craspedotella

\begin{tabular}{lccccc}
\hline Taxon & Latitud & Longitud & Prof. & Diám. & Figura \\
\hline Craspedotella sp. & $33^{\circ} 21^{\prime} \mathrm{S}$ & $78^{\circ} 06^{\prime} \mathrm{W}$ & 60 & 132 & $1,2,3$ \\
Craspedotella cf. pileous & $8^{\circ} 23^{\prime} \mathrm{S}$ & $141^{\circ} 14^{\prime} \mathrm{W}$ & 40 & 52 & $4,5,6$ \\
cf. Craspedotella (cónica aplanada con espina apical) & $13^{\circ} 32^{\prime} \mathrm{S}$ & $132^{\circ} 07^{\prime} \mathrm{W}$ & 100 & 40 & 7 \\
cf. Craspedotella (cónica con espina apical) & $20^{\circ} 27^{\prime} \mathrm{S}$ & $122^{\circ} 53^{\prime} \mathrm{W}$ & 190 & 57 & 8,9 \\
cf. Craspedotella (cónica con espina apical) & $30^{\circ} 30^{\prime} \mathrm{N}$ & $138^{\circ} \mathrm{E}$ & 200 & 73 & 10 \\
cf. Craspedotella (cónica con espina apical) & $30^{\circ} 02^{\prime} \mathrm{S}$ & $98^{\circ} 23^{\prime} \mathrm{W}$ & 110 & 76 & $11,12,13$ \\
\hline
\end{tabular}



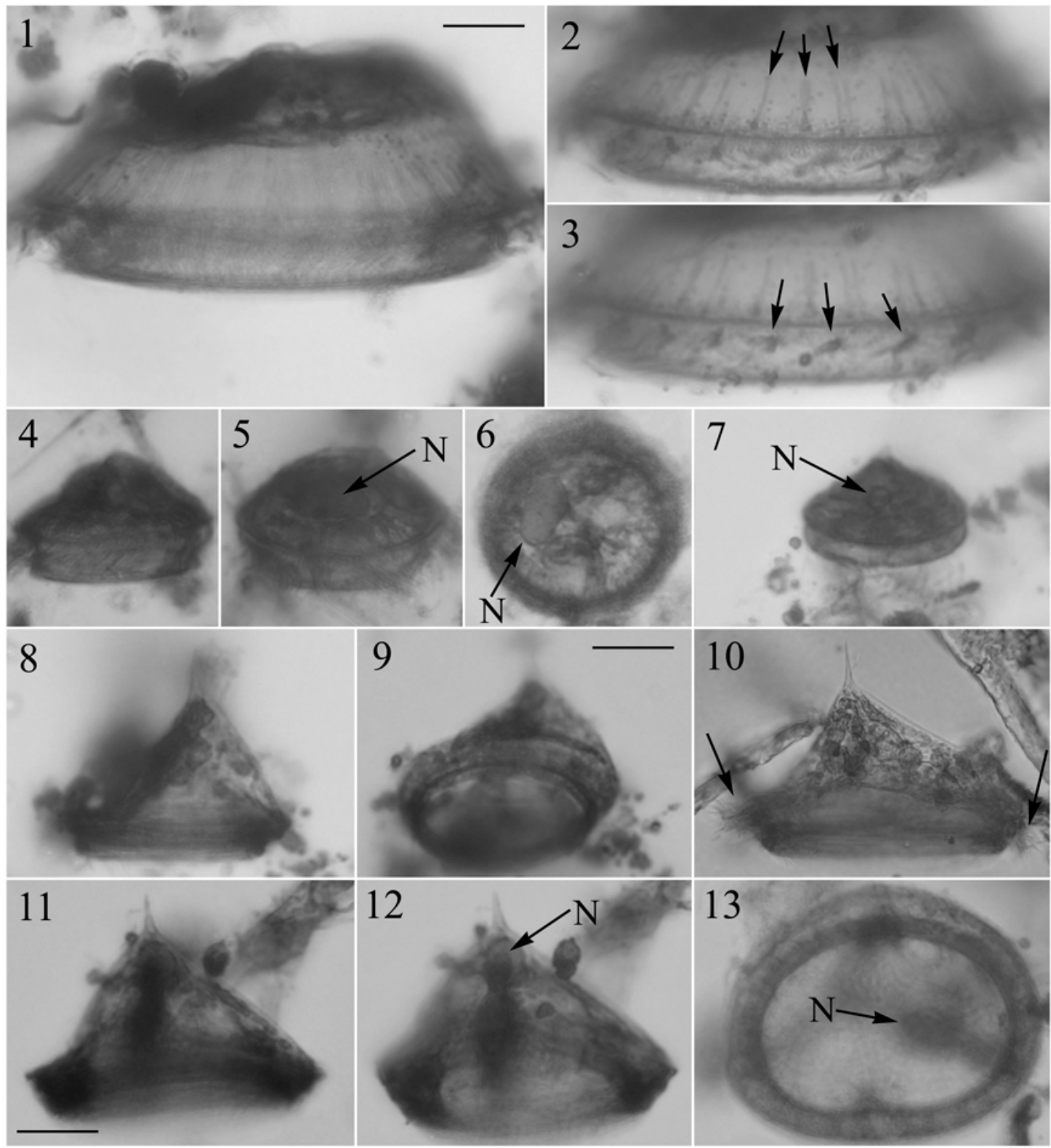

Figuras 1-13

Foto micrografías de especímenes atribuidos al género Craspedotella. (1-3) Craspedotella sp. Las flechas en figura 2 señalan las fibrillas radiales en la parte inferior del domo. Las flechas en la figura 3 señalan unos engrosamientos oblicuos de naturaleza desconocida en la parte central de la banda. (4-6) Craspedotella cf. pileolus. (7-13) Células cónicas que se asemejan a la forma de Craspedotella. (10) Las flechas señalan vellosidades que pueden corresponder a cilios. Todas las fotografías a la misma escala. $\mathbf{N}=$ Núcleo. Barra de escala de $20 \mu \mathrm{m}$

Photomicrographs of the specimens assigned to Craspedotella. (1-3) Craspedotella sp. The arrows in the fig. 2 indicate the radial fibrils in the lower margin of the dome. The arrows in the fig. 3 indicate structures of unknown nature in the central part of the band. (4-6) Craspedotella cf. pileolus (7-13) Conical cells that resemble the shape of Craspedotella. (10) The arrows indicate "hairs" that may correspond to ciliary structures. All the micrographs at the same scale. $N=$ Nucleus. Scale bar of $20 \mu \mathrm{m}$ 

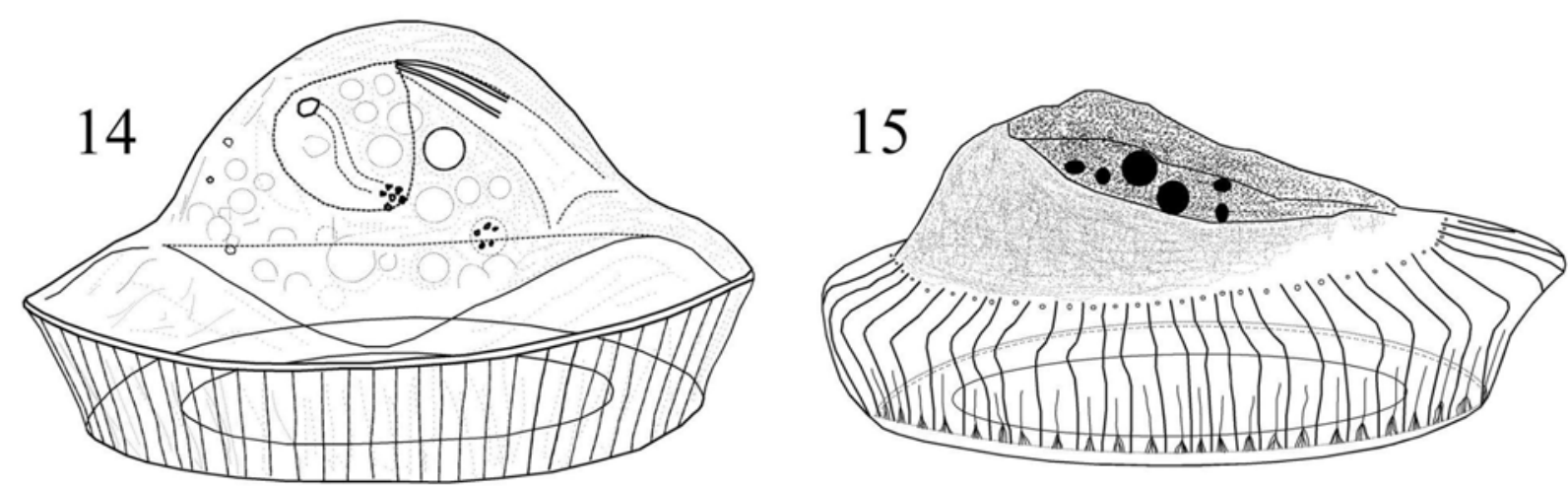

Figuras 14-15

Craspedotella pileolus (14) en Chatton (1952, pág. 342) y (15) en Cachon \& Cachon (1969)

Craspedotella pileolus (14) in Chatton (1952, p. 342) and (15) in Cachon \& Cachon (1969)

En este estudio se describen especímenes que se aproximan a la descripción de Craspedotella. Las dificultades en la precisa identificación son debidas a la falta de ilustración en la descripción original de Kofoid (1905) y sus trabajos posteriores. En una publicación póstuma de E. Chatton se reproduce una ilustración de C. pileolus que en su legenda se atribuye a C. A. Kofoid, sin dar más detalles sobre su origen (Chatton, 1952). La célula muestra un domo semiesférico con numerosas vacuolas y con dos vainas de flagelo y un citostoma en su parte superior. El domo se extiende sobre una banda con fibrillas transversales. La base de la célula presenta un orificio circular (Fig. 14). Las ilustraciones de C. pileolus por Cachon \& Cachon (1969) son detalladas (Fig. 15) e incluso incluyen una fotografía en Sournia (1986, pág. 165). Cachon \& Cachon (1969) no muestran la presencia de flagelos ni una banda inferior bien marcada. Unas fibrillas se extienden desde la región del citostoma hasta la base de la célula y entre estas fibrillas aparecen otras más cortas y ramificadas en su base (Fig. 15). A pesar de las diferencias con respecto a la descripción original, Cachon \& Cachon (1969) atribuyen sus especímenes mediterráneos a la especie tipo. Kofoid (1905) describe Craspedotella con un diámetro de 150-180 $\mu \mathrm{m}$, mientras que los especímenes de Cachon \& Cachon no exceden de $120 \mu \mathrm{m}$. En este caso, el tamaño no es un buen criterio para la separación de especies debido a los cambios de forma de las leptodiscáceas cuya locomoción se basa en la contracción y expansión del cuerpo celular (Cachon \& Cachon 1969). También deben tenerse en cuenta las dificultades a la hora de calibrar las medidas de tamaño en esos primeros estudios como Kofoid (1905).

En los especímenes atribuidos al género Craspedotella en este estudio no se ha podido observar la presencia de flagelos, que sin embargo eran visibles en otros géneros de leptodiscáceas procedentes de las mismas muestras (Gómez \& Furuya 2004, 2005). Tanto en Kofoid (1905) como en Cachon \& Cachon (1969) las fibrillas se extienden hasta la base de la células, mientras que en el espécimen de la figura 1-3 sólo se extienden desde el citostoma hasta el límite superior de la banda. Las fibrillas con ramificaciones en su base (Fig. 2) se asemejan a éstas en Craspedotella sensu Cachon \& Cachon (Fig. 15). En la zona media de la banda aparecen unas estructuras de naturaleza desconocida, tentativamente poros, entre cada dos fibrillas (Fig. 3) y que no aparecen en las ilustraciones de Cachon \& Cachon (1969). La forma del espécimen ilustrado en las figuras 4-6 se asemeja a Craspedotella sensu Kofoid. Los otros especimenes presentan una forma cónica con una espina más o menos desarrollada en el extremo superior del domo (Figs. 7-13). Esos especímenes no se ajustan a la descripción de $C$. pileolus, ni de Kofoid, ni de Cachon \& Cachon. Unas vellosidades que se observan en la región de la banda podrían corresponder a cilios (Fig. 10).

Más de un siglo después de la descripción del género Craspedotella es aún confusa su morfología. 
Este estudio ilustra por primera vez especímenes en el Pacífico que podrían corresponder a este género, quedando a la espera de nuevos estudios que aclaren las dudas en su identidad.

\section{Agradecimientos}

Este estudio es una contribución al proyecto BIOSOPE del LEFE-CYBER. Mi agradecimiento a J. Ras por su asistencia en la recogida de muestras y a los evaluadores por sus comentarios y sugerencias

\section{Literature cited}

Cachon J \& M Cachon. 1969. Contribution à l'étude des Noctilucidae Saville-Kent. Évolution morphologique, cytologie, systématique. II. - Les Leptodiscinae Cachon J. et M. Protistologica 5: 11-33.

Chatton E. 1952. Classe des Dinoflagellés ou Péridiniens. En: Grassé PP (ed). Traité de Zoologie, pp. 309-406. Masson, París.
Gómez F \& K Furuya. 2004. New records of Scaphodinium mirabile (Dinophyceae), an unnoticed dinoflagellate in the Pacific Ocean. Phycological Research 52: 13-16.

Gómez F \& K Furuya. 2005. Leptodiscaceans (Noctilucales, Dinophyceae) from the Pacific Ocean: First records of Petalodinium and Leptodiscus beyond the Mediterranean Sea. European Journal of Protistology 41: 231-239.

Kofoid CA. 1905. Craspedotella, a new genus of the Cystoflagellata, an example of convergence. Bulletin of the Museum of Comparative Zoology, Harvard 46 (9): 163-165.

Kofoid CA \& O Swezy. 1921. The free-living unarmoured Dinoflagellata. University of California, Memoirs 5: 1562.

Sournia A. 1986. Atlas du Phytoplancton Marin. Vol. I, 219 pp. Introduction, Cyanophycées, Dictyochophycées, Dinophycées et Raphidophycées. Editions du CNRS. París.

Recibido el 8 de noviembre de 2006 y aceptado el 8 de marzo de 2007 